\section{Endoscopic visualisation to aid deep anterior lamellar keratoplasty}

${ }^{1}$ Department of Ophthalmology Queen's University Belfast, Ireland

${ }^{2}$ Department of Ophthalmology Bedford Hospital Bedford, UK

Correspondence:

A Sharma

Department of

Ophthalmology

Bedford Hospital (South

Wing)

Bedfordshire MK42 9DJ, UK

E-mail: anant.sharma@

bedhos.anglox.nhs.uk

Received: 25 October 2002 Accepted in revised form: 26 March 2003

\begin{abstract}
Aims The aim of this study was to assess the value of endoscopy during deep anterior lamellar keratoplasty (DALK) by visualising the posterior cornea. This allows the surgeon to determine whether air injection had succeeded in stripping Descemet's membrane and endothelium from the posterior corneal stroma.

Methods Four whole globes for research were obtained from the Florida eye bank with consent. A $2 \mathrm{~mm}$ incision was placed at the limbus and the endoscope was introduced through this into the anterior chamber. A 26-gauge needle was introduced into the cornea with the bevel positioned as deep as possible and air injected into the corneal stroma. Air was injected until the whole cornea became opaque and repeated air injections were made even after an opaque cornea was noted. The endoscopic camera was used to visualise the posterior corneal surface during this procedure.

Results The view of the posterior corneal surface was clear and introduction of the probe did not interfere with the air dissection. In all four eyes, despite ease of air injection and diffuse corneal air infiltration, no large air bubble dissection of Descemet's membrane from adjacent stroma occurred. Instead multiple blistering of the posterior corneal surface could be seen.

Conclusions Endoscopy provides an effective tool to visualise the posterior corneal surface during DALK, using air dissection. This technique may become a standard adjunctive procedure during DALK.

Eye (2004) 18, 188-191. doi:10.1038/

sj.eye. 6700601
\end{abstract}

Keywords: endoscopic; keratoplasty; DALK; air dissection
JE Moore ${ }^{1,2}$, G Herath ${ }^{2}$ and A Sharma²

\section{Introduction}

Air dissection may be used as an operative tool while performing deep anterior lamellar keratoplasty (DALK). ${ }^{1-3}$ As described by Anwar and Teichmann, ${ }^{1}$ the optimum achievable scenario is when air separates Descemet's membrane from posterior corneal stroma, thus creating a space or 'bubble'. This end point can be difficult to ascertain if the overlying cornea is opaque due to the air dissection. Anwar and Teichmann ${ }^{1}$ stated that complete air infiltration of the cornea with a characteristic 'large bubble' appearance and 'ease of injection' were good indicators of successful air bubble formation at the Descemet's-stromal interface. ${ }^{1}$ Dissection without an air bubble can be time-consuming and can result in a more 'irregular' dissection with a less-than-optimal visual result.

The aim of this study was to visualise the posterior surface of the cornea with an ocular endoscope (Litechnica) introduced into the anterior chamber. This hopefully would allow definitive surgical decisions to be made based on whether adequate separation of Descemet's membrane from posterior corneal stroma had taken place during air dissection.

\section{Materials and methods}

Four whole globes for research were obtained from the Florida eye bank with consent. A $2 \mathrm{~mm}$ incision was placed at the limbus and the endoscope was introduced through this into the anterior chamber. The endoscope used is an 'Endo Optiks microendoscope' (Figure 1), which combines illumination and video imaging through a 20-gauge probe. The endoscope produces an image of 10000 pixels with a $110^{\circ}$ field of view and a range of focus from $0.5 \mathrm{~mm}$ to infinity.

The endoscope tip was positioned just beyond the 'lip' of the $2 \mathrm{~mm}$ section inside the anterior chamber. All procedures were carried 


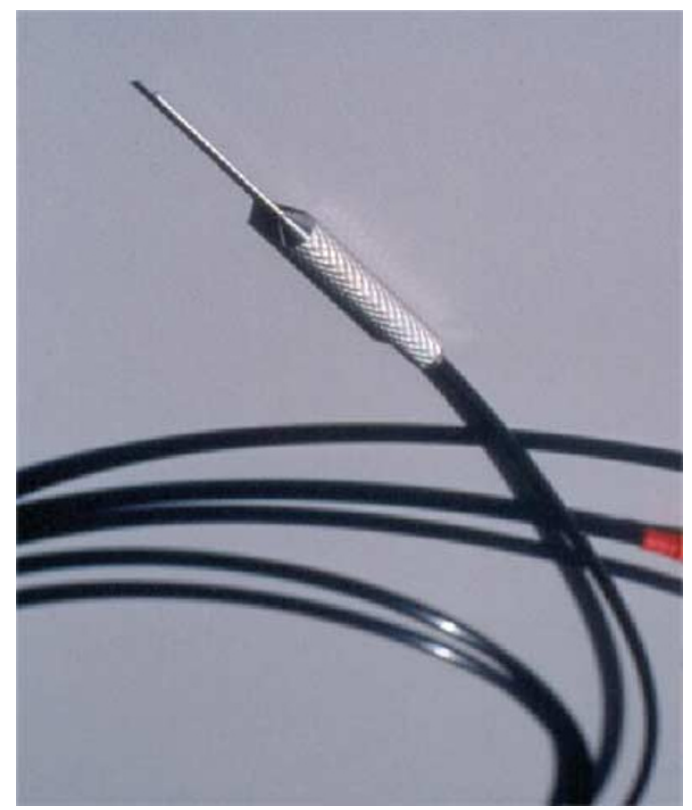

Figure 1 The endoscopic light probe (Endo Optiks microendoscope) combines illumination and video imaging through a 20gauge probe. The 10000 pixel endoscope has a $110^{\circ}$ field of view and the endoscope can be focused from $0.5 \mathrm{~mm}$ to infinity.

out with an assistant holding the endoscope to provide the surgical view; this enabled the surgeon 'free' use of both hands to manipulate intraocular instruments. The anterior chamber if required was filled with balanced salt solution (Alcon). A 26-gauge needle was introduced into the cornea with the bevel down and positioned as deep as possible and air injected into the corneal stroma (Figure 2). Air in the anterior chamber was replaced with BSS if the posterior corneal view was poor. In one of the four eyes, viscoelastic (Provisc, Alcon) was used to fill partly the anterior chamber in an attempt to slow down the entry of air into the anterior chamber during the air dissection of the cornea. This helped in visualising the point of entry of air into the anterior chamber.

Air was injected until the whole cornea became opaque and subsequent repeated air injections were made even after an opaque cornea was noted. The endoscope was used to visualise the posterior corneal surface during this procedure.

\section{Results}

Visualisation of the posterior corneal surface with the endoscope was clear and introduction of the probe did not interfere with the air dissection. In all four eyes, despite ease of air injection and diffuse corneal air infiltration, no 'large air bubble' dissection of Descemet's

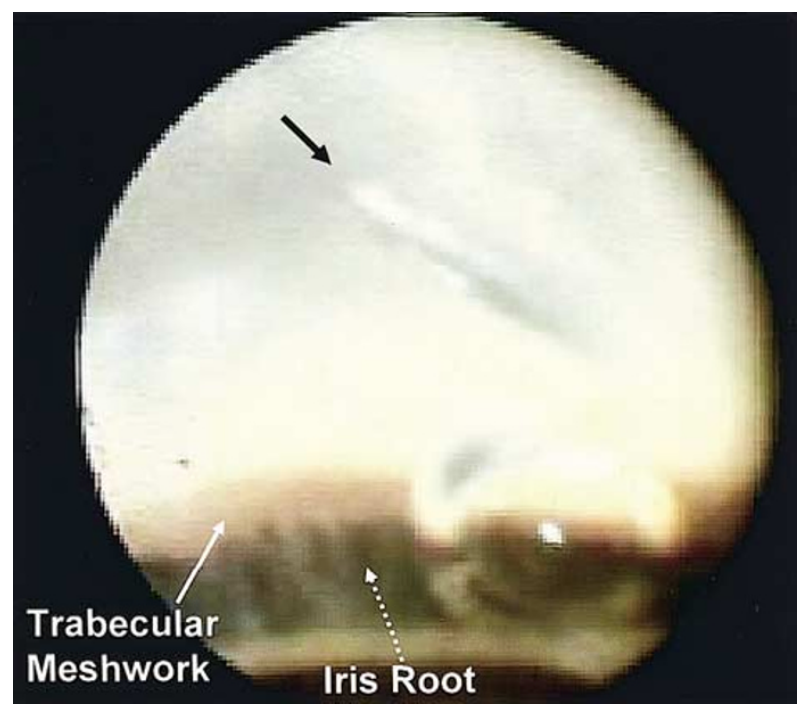

Figure 2 An endoscope was introduced through a $2 \mathrm{~mm}$ incision made at the limbus. A 26-gauge needle was then inserted into the corneal stroma bevel down (indicated with a black arrow). Its depth and proximity to Descemet's membrane could be viewed via the endoscopic camera from within the anterior chamber.

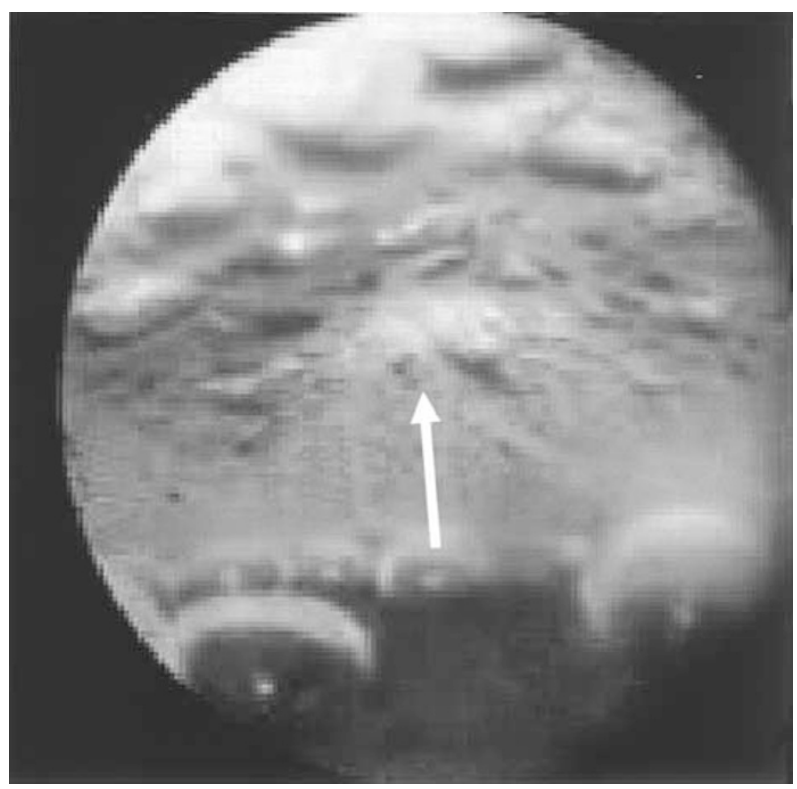

Figure 3 Air forcibly injected into the posterior corneal stroma did not produce a large 'bubble' dissection of Descemet's membrane from posterior stroma. Instead, multiple small air loculations were formed within the posterior stroma as indicated by the arrow.

membrane from adjacent stroma occurred. Instead, multiple blistering of the postcorneal surface could be seen (Figure 3). 


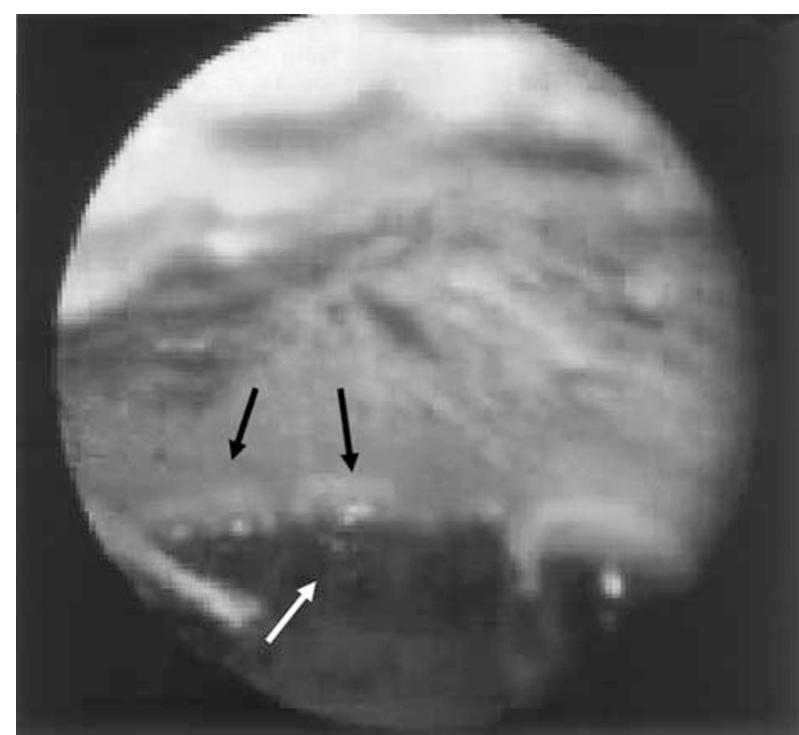

Figure 4 After intracorneal stroma air injection, air entered the anterior chamber either through the trabeculum or at the junction of peripheral cornea and the trabecular meshwork. As indicated by black arrows, air bubbles first appear at this site. Iris root is indicated with white arrow.

Repeat air injection did not produce a 'large air bubble' dissection. Interestingly, the air entry into the anterior chamber during the air injection appeared to be from the peripheral cornea or anterior trabecular meshwork (Figure 4).

\section{Discussion}

This study demonstrated the value of endoscopy to visualise the posterior corneal surface when the anterior cornea is opaque. No significant large air bubble, separating posterior corneal stroma from Descemet's membrane, was seen in any of the four cadaver eyes studied despite fulfilling previously published criteria indicative of successful air dissection. ${ }^{1}$ Potential reasons for poor air dissection in these cadaver eyes may be related to the period of time between harvesting and usage. However, a decompensated cornea in the cadaver eye, may allow an easier air dissection of Descemet's membrane form posterior corneal stroma.

It would appear from this study that formation of an adequate air bubble using air dissection to perform DALK is not commonly seen. Hence, it may be appropriate in the future to adopt the use of an endoscope to assess if the end point air bubble has been achieved. This would enable the surgeon to either continue with surgery, repeat dissection (with air or saline) or to change/abandon the technique. In this study, we did not find that repeat air dissection, even when the criteria for 'ideal dissection' were reached, helped in the formation of an adequate air bubble.

Posterior corneal techniques such as posterior lamellar keratoplasty (PLK) ${ }^{4,5}$ and Descemet's membrane endothelial transplantation ${ }^{6,7}$ are at present still being developed. In such transplant situations, the cornea will be more opaque and viewing the posterior cornea will be more difficult. Endoscopy may aid such techniques especially where Descemet's membrane and endothelial transplantation is being attempted. We are currently investigating the use of endoscopy to aid such techniques.

Endoscopy is a useful tool to visualise structures inside the eye if the direct surgical microscopic view is obscured, for example, due to media opacification. It has been used for a variety of clinical situations to view structures such as the ciliary body directly and accurately. This technique has potential to assist surgery in any case where the view is obscured posteriorly due to opaque intervening structures such as corneal and lens opacification or vitreous haemorrhage. 8,9

Air-bubble dissection is a valuable method used in DALK surgery; we feel that endoscopy may become an essential part of the armament of tools to be used by surgeons for this type of procedure. In addition, further advances in endoscopy such as improved endoscopic design allowing stereoscopy ${ }^{10-12}$ may greatly increase the potential for this technique.

\section{References}

1 Anwar M, Teichmann KD. Big-bubble technique to bare Descemet's membrane in anterior lamellar keratoplasty. J Cataract Refract Surg 2002; 28(3): 398-403.

2 Archila EA. Deep lamellar keratoplasty dissection of host tissue with intrastromal air injection. Cornea 1984; 3: 217-218.

3 Chau GK, Dilly SA, Sheard CE, Rostron CK. Deep lamellar keratoplasty on air with lyophilised tissue. Br J Ophthalmol 1992; 76: 646-650.

4 Melles GR. Posterior lamellar keratoplasty. Arch Soc Esp Oftalmol 2002; 77: 175-176.

5 Melles GR, Lander F, Nieuwendaal C. Sutureless, posterior lamellar keratoplasty: a case report of a modified technique. Cornea 2002; 21: 325-327.

6 Melles GR, Lander F, Rietveld FJ. Transplantation of Descemet's membrane carrying viable endothelium through a small scleral incision. Cornea 2002; 21: 415-418.

7 Sharma A, Woodman A. Comment on transplantation of Descemet's membrane carrying viable endothelium through a small scleral incision. Cornea 2000; 21(8): 840. 
8 Joos KM, Shen JH. An ocular endoscope enables a goniotomy despite a cloudy cornea. Arch Ophthalmol 2001; 119: 134-135.

9 Ciardella AP, Fisher YL, Carvalho C et al. Endoscopic vitreoretinal surgery for complicated proliferative diabetic retinopathy. Retina 2001; 21: 20-27.

10 Miyake K, Ota I, Miyake S, Tanioka K,

Kubota M, Mochizuki R. Application of a newly developed, highly sensitive camera and a 3-dimensional high-definition television system in experimental ophthalmic surgeries. Arch Ophthalmol 1999; 117: 1623-1629.

11 van Bergen P, Kunert W, Buess GF. Three-dimensional (3-D) video systems: bi-channel or single-channel optics? Endoscopy 1999; 3: 732-737.

12 Yao K, Matsui T, Furukawa H, Yao T, Sakurai T, Mitsuyasu T. A new stereoscopic endoscopy system: accurate 3-dimensional measurement in vitro and in vivo with distortion-correction function. Gastrointest Endosc 2002; 55: 412-420. 